EINDHOVEN UNIVERSITY OF TECHNOLOGY

Department of Mathematics and Computing Science

Memorandum COSOR 94-24

Infinite divisibility and the waiting-time paradox

K. van Harn

F.W. Steutel

Eindhoven, August 1994

The Netherlands 


\title{
INFINITE DIVISIBILITY AND THE WAITING-TIME PARADOX
}

\author{
K. VAN HARN AND F.W. STEUTEL
}

\begin{abstract}
It is shown that the interarrival time $Z$ covering the point zero in a stationary renewal process generated by $X$ has the form $Z \stackrel{\mathrm{d}}{=} X+Y$ with $Y$ nonnegative and independent of $X$, if and only if $X$ is infinitely divisible. In the special case that $X$ has a compound-exponential distribution there is a similar decomposition of the stationary waiting time. These results shed some new light on the waiting-time paradox.
\end{abstract}

\section{Introduction and summary}

The waiting-time paradox consists of the fact that a passenger arriving at a bus stop will probably have to wait considerably longer than about half the interarrival time, $X$ say, of two buses. The paradox is resolved by the observation that a passenger is more likely to arrive in a long interval than in a short one. Alternatively, the length $Z$ of the interval covering the arrival time of the passenger tends to be longer than $X$, i.e., we have

$$
Z \geq X
$$

in distribution. On an appropriate sample space (1.1) can be written as

$$
Z \stackrel{\mathrm{d}}{=} X+Y
$$

where $Y$ is nonnegative and, in general, $X$ and $Y$ are dependent.

In this note we consider the case where $X$ and $Y$ in (1.2) are independent. It turns out that this happens if and only if $X$ is infinitely divisible, and this result sheds some new light on the waiting-time paradox and on the behaviour of nonnegative processes with stationary, independent increments [sii-processes]. In the special case that $X$ is compound-exponential [ see Definition 2.5], also the (stationary) waiting-time $W$ of a bus passenger admits of a decomposition similar to (1.2):

$$
W \stackrel{\mathrm{d}}{=} X+A,
$$

where $A$ is nonnegative and independent of $X$.

In Section 2 we collect some results about renewal theory and infinite divisibility. Section 3 contains a characterization of infinite divisibility for nonnegative random variables by means of (1.2), and some of its consequences. In Section 4 equation (1.3) is considered for non-lattice distributions. In Section 5 we briefly present the solutions of (1.2) and (1.3) for distributions on the non-negative integers. We shall mostly use the renewal (life-time) terminology rather than the waiting-time terminology. 


\section{Preliminaries}

We need some information on renewal theory and on infinite divisibility for nonnegative random variables. Some of the results hold only for non-lattice distributions, and have analogues for lattice distributions. We shall only consider lattice random variables with values in $\mathbb{Z}_{+}:=\mathbb{N} \cup\{0\}$. Sometimes it will be essential that these random variables have positive probability at 0 .

\subsection{Renewal theory}

We consider a renewal process generated by a sequence $\left(X_{n}\right)_{n \in \mathrm{N}}$ of independent, non-negative random variables distributed as $X$ with distribution function $F$ and expectation $\mathbb{E} X=\mu \in(0, \infty)$; these conditions will be assumed to hold throughout unless otherwise stated. We write $S_{n}=\sum_{k=1}^{n} X_{k}$, and define the number $N_{t}$ of renewals in $(0, t]$, the age $V_{t}$ of the unit in service at time $t$, the remaining lifetime $W_{t}$ of this unit (the waiting time for the next bus), and the total life-time $Z_{t}$ as follows:

$$
\begin{aligned}
& N_{t}:=\#\left(\left\{n \in \mathbb{N}: S_{n} \leq t\right\}\right), \\
& V_{t}:=t-S_{N_{t}}, \quad W_{t}:=S_{N_{t}+1}-t, \\
& Z_{t}=X_{N_{t}+1}=V_{t}+W_{t}
\end{aligned}
$$

The following result is well known; see Feller (1971).

Lemma 2.1. If $F$ is non-lattice, then

$$
V_{t} \stackrel{\mathrm{d}}{\longrightarrow} V, W_{t} \stackrel{\mathrm{d}}{\longrightarrow} W \stackrel{\mathrm{d}}{=} V, Z_{t} \stackrel{\mathrm{d}}{\rightarrow} Z \quad[t \rightarrow \infty],
$$

where $Z$ and $W$ have distribution functions given by

$$
\begin{aligned}
& F_{Z}(z)=\frac{1}{\mu} \int_{(0, z]} x d F(x) \quad[z \geq 0] \\
& F_{W}(w)=\frac{1}{\mu} \int_{0}^{w}\{1-F(x)\} d x \quad[w \geq 0] .
\end{aligned}
$$

Alternatively, $V, W$ and $Z$ can be regarded as quantities in a stationary renewal process (started at $-\infty$ ); then $Z$ is the life time straddling 0 , say, and $V$ and $W$ are the lengths of the parts into which $Z$ is divided by 0 . The random variables $V, W$ and $Z$ will be used in this sense, sometimes without comment. They satisfy

$$
Z=V+W \text { and } V \stackrel{\mathrm{d}}{=} W \stackrel{\mathrm{d}}{=} U Z,
$$

where $U$ is uniformly distributed on $(0,1)$ and independent of $Z$; see Winter $(1989)$. 
There is an analogue to Lemma 2.1 for lattice distributions. Now $X$ is distributed on $\mathbb{Z}_{+}$, and the quantities $N_{n}, V_{n}, W_{n}$ and $Z_{n}$ are defined for $n \in \mathbb{N}$ in the same way as $N_{t}, V_{t}, W_{t}$ and $Z_{t}$. We then have the following well-known result; see Feller (1968). We use the notations $V, W$ and $Z$ also for the limits of $V_{n}, W_{n}$ and $Z_{n}$.

Lemma 2.2. If $X$ has an aperiodic distribution $\left(p_{k}\right)_{k \in \mathbb{Z}_{+}}$on $\mathbb{Z}_{+}$, then

$$
V_{n} \stackrel{\mathrm{d}}{\longrightarrow} V, W_{n} \stackrel{\mathrm{d}}{\longrightarrow} W \stackrel{\mathrm{d}}{=} V+1, Z_{n} \stackrel{\mathrm{d}}{\longrightarrow} Z \quad[n \rightarrow \infty],
$$

where $Z$ and $W$ have distributions given by

$$
\begin{aligned}
& \mathbb{P}(Z=k)=\frac{1}{\mu} k p_{k} \quad[k \in \mathbb{N}], \\
& \mathbb{P}(W=k)=\frac{1}{\mu} \sum_{j=k}^{\infty} p_{j} \quad[k \in \mathbb{N}] .
\end{aligned}
$$

Remark. Comparison with (2.4) learns that (2.1) also holds for distributions on $\mathbb{Z}_{+}$. Since the span of the lattice is of no real importance, $(2.1)$ holds for all distributions on $\mathbb{R}_{+}$with a finite moment. Since $W$ in (2.2) is absolutely continuous, the non-lattice condition there is essential.

\subsection{Infinite divisibility}

We recall the definition of infinite divisibility: a random variable $X$ (or its distribution function $F$ ) is said to be infinitely divisible if for any $n \in \mathbb{N}$ iid random variables $X_{1}^{(n)}, \ldots, X_{n}^{(n)}$ exist such that

$$
X \stackrel{\mathrm{d}}{=} X_{1}^{(n)}+\cdots+X_{n}^{(n)} .
$$

In what follows the Laplace-Stieltjes transform [LSt] of a function $H$ will be denoted by $\widehat{H}$. We take the following result from Feller (1971).

Lemma 2.3. A distribution function $F$ on $\mathbb{R}_{+}$is infinitely divisible iff its LSt satisfies

$$
\log \widehat{F}(s)=\int_{0}^{\infty}\left(e^{-s x}-1\right) x^{-1} d K(x),
$$

where $K$ is a nondecreasing function on $\mathbb{R}_{+}$with (necessarily) $\int_{1}^{\infty} x^{-1} d K(x)<\infty$.

$K$ will be called the canonical function of $F$, or of the corresponding random variable. We note that the random variables $X^{(n)}$ in (2.6) are infinitely divisible with distribution function $F^{*(1 / n)}$, the $n$-th convolution root of $F$, and with canonical function $K^{(n)}=(1 / n) K$. Differentiation of $(2.7)$ yields

$$
-\widehat{F}^{\prime}(s) / \widehat{F}(s)=\widehat{K}(s),
$$

and inversion of this leads to the following equivalent relation [see Steutel(1970)]; let $s \downarrow 0$ for the final statement. 
Corollary 2.4. A distribution function $F$ on $\mathbb{R}_{+}$is infinitely divisible iff $F$ satisfies

$$
\int_{(0, x]} y d F(y)=(F * K)(x) \quad[x>0],
$$

where $K$ is nondecreasing on $\mathbb{R}_{+}$and * denotes convolution. The, possibly infinite, first moment $\mu$ of $F$ is given by

$$
\mu=\int_{[0, \infty)} d K(x)=K(\infty)
$$

For Section 4 we need the following subclass of infinitely divisible distributions.

Definition 2.5. Let $S(\cdot)$ be a process with stationary, independent increments and let $T$ be exponentially distributed with $\mathbb{E} T=1$, independent of $S(\cdot)$. Then the random variable $X:=S(T)$ is said to have a compound-exponential distribution.

The following lemma is immediate if we denote the (infinitely divisible) distribution function of $S(1)$ by $F_{0}$.

Lemma 2.6. $A$ distribution function $F$ on $\mathbb{R}_{+}$is compound-exponential iff $\hat{F}$ has the form

$$
\widehat{F}(s)=\frac{1}{1-\log \widehat{F}_{0}(s)},
$$

where $F_{0}$ is an infinitely divisible distribution function on $\mathbb{R}_{+}$. The distribution functions $F$ and $F_{0}$ have the same first moment.

We conclude this section with analogues (and also special cases) of Corollary 2.4 and Lemma 2.6 for infinitely divisible distributions on $\mathbb{Z}_{+}$. Here we use probability generating functions [pgf's] rather then LSt's. We stress hat the factors $X_{j}^{(n)}$ in (2.3) are $\mathbb{Z}_{+}$-valued iff $P(X=0)>0$; for details we refer to Steutel (1970).

Lemma 2.7. A distribution $\left(p_{k}\right)_{k \in \mathbb{Z}_{+}}$on $\mathbb{Z}_{+}$with $p_{0}>0$ is infinitely divisible iff the quantities $r_{k}$ with $k \in \mathbb{Z}_{+}$defined by

$$
(k+1) p_{k+1}=\sum_{j=0}^{k} p_{j} r_{k-j} \quad\left[k \in \mathbb{Z}_{+}\right],
$$

are nonnegative, i.e., iff the coefficients of $R(z):=P^{\prime}(z) / P(z)$ are nonnegative.

Lemma 2.8. $A$ distribution on $\mathbb{Z}_{+}$with pgf $P$ is compound-exponential iff $P$ has the form

$$
P(z)=\frac{1}{1-\log Q(z)}=\frac{1}{1-\alpha(G(z)-1)},
$$

where $Q$ is the pgf of an infinitely divisible distribution on $\mathbb{Z}_{+}$with $Q^{\prime} / Q=\alpha G^{\prime}$, $\alpha>0$ and $G$ a pgf。 


\section{An extreme case of the waiting-time paradox}

We return to the renewal process generated by a nonnegative random variable $X$ as described in Section 2.1. It is well known [see e.g. Ross (1970)] that for the total life-time $Z$ with distribution function given by (2.1) we have

$$
Z \stackrel{d}{\geq} X
$$

i.e., $\mathbb{P}(Z>x) \geq \mathbb{P}(X>x)$ for $x \in \mathbb{R}_{+}$, or on a suitable sample space,

$$
Z \stackrel{\mathrm{d}}{=} X+Y
$$

where $Y$ is nonnegative and, in general, not independent of $X$. Here we are interested in the situation where $X$ and $Y$ are independent. The following theorem can also be read as a characterization theorem for infinitely divisible random variables on $\mathbb{R}_{+}$ with a finite first moment [compare Remark following Lemma 2.2].

Theorem 3.1. Let $Z$ be the life time covering the point 0 in a stationary renewal process generated by $X$. Then $Z$ can be written as in (3.1) with $X$ and $Y$ independent iff $X$ is infinitely divisible. The distribution function of $Y$ is given by $F_{Y}=(1 / \mu) K$, where $K$ is the canonical function of $X$.

Proof. Let $Z$ satisfy (3.1) with $X$ and $Y$ independent. Then by (2.1) we have

$$
F_{Z}(z)=\frac{1}{\mu} \int_{(0, z]} x \mathrm{~d} F(x)=\left(F * F_{Y}\right)(z) \quad[z \geq 0] .
$$

Multiplying by $\mu$ we see that $F$ satisfies the functional equation (2.9) with $K=\mu F_{Y}$, hence by Corollary $2.4 F$ is infinitely divisible. Conversely, if $F$ is infinitely divisible, then by (2.10) the functional equation (2.9) can be written in the form (3.2) for some distribution function $F_{Y}$, which means that in (3.1) $X$ and $Y$ can be taken independent.

Remark. By a suitable choice of $K$, for $F_{Y}=(1 / \mu) K$ any distribution function is possible. If $F(0)>0$, then $X$ is compound-Poisson [cf. van Harn (1978)], i.e.,

$$
X \stackrel{\mathrm{d}}{=} \widetilde{X}_{1}+\cdots+\widetilde{X}_{N}
$$

where $\widetilde{X}_{1}, \widetilde{X}_{2}, \ldots$ are independent and distributed as $\tilde{X}$ with distribution function $G$, say, and $N$ is Poisson distributed, independent of the $\widetilde{X}_{j}$ 's. Now $F_{Y}$ takes the form

$$
F_{Y}(y)=\frac{1}{\mathbb{E} \tilde{X}} \int_{(0, y]} u \mathrm{~d} G(u)
$$

i.e., $Y$ has the same distribution as the total life-time $\widetilde{Z}$ in the renewal process generated by $\tilde{X}$. This can, roughly, be explained as follows. Since $X$ is a Poisson sum 
of $\widetilde{X}_{j}$ 's, the (positive) $X$-interval covering a fixed point can be regarded as the sum of the $\widetilde{X}$-interval covering this point and a (Poisson) number of independent $\widetilde{X}_{j}$ 's, i.e., as $X+\widetilde{Z} \stackrel{\text { d }}{=} X+Y$.

The question remaining is: what is the meaning of the random variable $Y$ in (3.2). An answer can be obtained by considering the renewal process generated by $X^{(n)}$ with distribution function $F^{*(1 / n)}$. Denoting the corresponding $Z$-random variable by $Z^{(n)}$, and using the fact that $X^{(n)}$ is infinitely divisible with canonical function $K^{(n)}=(1 / n) K$, by Theorem 3.1 we have

$$
Z^{(n)} \stackrel{\mathrm{d}}{=} X^{(n)}+Y^{(n)},
$$

where the distribution function of $Y^{(n)}$ is given by

$$
F_{Y(n)}=\frac{1}{\mathbb{E} X^{(n)}} K^{(n)}=\frac{n}{\mu} K^{(n)}=\frac{1}{\mu} K=F_{Y},
$$

independent of $n$. More generally, considering the sii-process $X(\cdot)$ with $X(1) \stackrel{\mathrm{d}}{=} X$ and $X(1 / n) \stackrel{\text { d }}{=} X^{(n)}$, we obtain the following result.

Theorem 3.2. Let $X$ be a nonnegative infinitely divisible random variable with finite first moment $\mu$ and with canonical function $K$. Let $X(\cdot)$ denote the sii-process with $X(1) \stackrel{\mathrm{d}}{=} X$, and for $t>0$ let $Z(t)$ be the $Z$-random variable in a stationary renewal process generated by $X(t)$. Then

$$
Z(t) \stackrel{\mathrm{d}}{=} X(t)+Y \quad[t>0]
$$

where $X(t)$ and $Y$ are independent and $F_{Y}=(1 / \mu) K$, independent of $t$.

Equation (3.3) tells us that, if we approach the point 0 with small steps, i.e., if $t$ is small, then this point is passed with a non-small step of length at least $Y$, in distribution. Also, though $X(t) \stackrel{\mathrm{d}}{\longrightarrow} 0$, we have $Z(t) \stackrel{\mathrm{d}}{\longrightarrow} Y \neq 0$ as $t \downarrow 0$ : we get an extreme case of the waiting-time paradox. For large $t$, on the other hand, the term $X(t)$ will dominate in the right-hand side of (3.3): the paradox, almost, disappears. Combining (2.3) with (3.3) yields the following result for the remaining life-time $W$.

Corollary 3.3. Let $X(\cdot)$ be as in Theorem 3.2, and for $t>0$ let $W(t)$ be the $W$ random variable in a stationary renewal process generated by $X(t)$. Then

$$
W(t) \stackrel{d}{=} U X(t)+U Y \quad[t>0]
$$

where $U, X(t)$ and $Y$ are independent, $U$ is uniform on $(0,1)$, and $Y$ is as in Theorem 3.2. In particular,

$$
W(t) \stackrel{\mathrm{d}}{\longrightarrow} U Y \quad[t \downarrow 0] .
$$




\section{Compound-exponential life times}

Since the compound-exponential distributions [cf. Definition 2.5] are a special subclass of the infinitely divisible distributions, one may expect the random variable $Y$ in (3.1) to have special properties, if $X$ is taken compound-exponential. Indeed we have the following result.

Theorem 4.1. If $X$ is a nonnegative, compound-exponential random variable with a finite first moment, then the life time $Z$ covering 0 in a stationary renewal process generated by $X$ satisfies

$$
Z \stackrel{\mathrm{d}}{=} X+X^{\prime}+Y_{0}
$$

where $X^{\prime} \stackrel{\mathrm{d}}{=} X, Y_{0}$ is nonnegative, and $X, X^{\prime}$ and $Y_{0}$ are independent. Equivalently, the random variable $Y$ in (3.1) satisfies $Y \stackrel{d}{=} X^{\prime}+Y_{0}$ with $X^{\prime}$ and $Y_{0}$ independent and independent of $X$.

Proof. By Lemma 2.6 the LSt $\widehat{F}$ of $X$ has the form $\widehat{F}(s)=1 /\left(1-\log \widehat{F}_{0}(s)\right)$, where $F_{0}$ is an infinitely divisible distribution function on $\mathbb{R}_{4}$. From this equation and (2.8) it follows that the canonical function $K$ of $F$ is related to the canonical function $K_{0}$ of $F_{0}$ by $K=F * K_{0}$ :

$$
\widehat{K}(s)=-\frac{\mathrm{d}}{\mathrm{d} s} \log \widehat{F}(s)=\widehat{F}(s)\left\{-\frac{\mathrm{d}}{\mathrm{d} s} \log \widehat{F}_{0}(s)\right\}=\widehat{F}(s) \widehat{K}_{0}(s) .
$$

Dividing by $\mu$, the first moment of both $F$ and $F_{0}$, and letting $Y_{0}$ be a random variable with distribution function $(1 / \mu) K_{0}$, we see that $Y$ in (3.1) satisfies

$$
F_{Y}=F * F_{Y_{0}}
$$

so $Y$ can be obtained as $Y \stackrel{\mathrm{d}}{=} X^{\prime}+Y_{0}$ with $X^{\prime}$ and $Y_{0}$ independent.

Remark. As in Theorem 3.2 one can consider the renewal process generated by $X(t)$. When $X=X(1)$ is compound-exponential, then so is $X(t)$ for $0<t<1$. Repeating the calculations for Theorem 4.1 with $X$ replaced by $X(t)$, we obtain

$$
Z(t) \stackrel{\mathrm{d}}{=} X(t)+X^{\prime}(t)+Y_{0}(t) \quad[0<t \leq 1]
$$

where $Y_{0}(t) \stackrel{\mathrm{d}}{=} X(1-t)+Y_{0}$ with $Y_{0}$ as in (4.1) independent of $X(1-t)$. So, we recover (3.3) with $Y \stackrel{\mathrm{d}}{=} X+Y_{0}$ and $Y_{0}$ as in (4.1).

Choosing a uniformly distributed random variable $U$ independent of $X, X^{\prime}$ and $Y_{0}$, we can rewrite (4.1) as

$$
Z \stackrel{\mathrm{d}}{=}\left(X+U Y_{0}\right)+\left(X^{\prime}+\bar{U} Y_{0}\right)
$$


where $\bar{U}=1-U \stackrel{\mathrm{d}}{=} U$. Since we also have $Z=V+W$ with $V \stackrel{\text { d }}{=} W[$ cf. Lemma 2.1 and (2.3)], this suggests that in this case

$$
W \stackrel{\mathrm{d}}{=} X+A
$$

where $X$ and $A$ are independent with $A$ of the form $A=U Y_{0}$. This is the content of the next theorem. Since apart from some mass at zero the random variable $A$, and hence $W$, has an absolutely continuous distribution, we consider the non-lattice case here; cf. Remark following Lemma 2.2.

Theorem 4.2. If $X$ is compound-exponential and non-lattice with finite first moment $\mu$, then the stationary remaining life-time $W$ satisfies (4.3) with $X$ and $A$ independent, and $A$ of the form $A \stackrel{\mathrm{d}}{=} U Y_{0}$, where $Y_{0}$ is nonnegative, $U$ is uniform on $(0,1)$ and independent of $Y_{0}$.

Proof. Use Lemmas 2.1, 2.3 and 2.6. Since $X$ has LSt $\widehat{F}=1 /\left(1-\log \widehat{F}_{0}\right)$ with $F_{0}$ infinitely divisible, the LSt of $W$ can be written as

$$
\widehat{F}_{W}(s)=\frac{1-\widehat{F}(s)}{\mu s}=\widehat{F}(s) \frac{-\log \widehat{F}_{0}(s)}{\mu s}=\widehat{F}(s) \int_{0}^{\infty} \frac{1-\mathrm{e}^{-s x}}{s x} \mathrm{~d} F_{Y_{0}}(x),
$$

where, as in the proof of Theorem 4.1, $F_{Y_{0}}=(1 / \mu) K_{0}$ with $K_{0}$ the canonical function of $F_{0}$. The equality in the extreme members of this equation easily translates in (4.3) with $A$ of the form $A=U Y_{0}$ as desired.

It is unclear whether (4.3) with $X$ and $A$ independent implies that $X$ has a compoundexponential distribution. The fact that $A$ is of the form $A \stackrel{\mathrm{d}}{=} U Y_{0}$ implies that $A$ has a density on $(0, \infty)$ which is nonincreasing.

Remark. Proceeding as in the Remark following Theorem 4.1, for $W(t)$ we obtain

$$
W(t) \stackrel{\mathrm{d}}{=} X(t)+U Y_{0}(t) \quad[0<t \leq 1]
$$

which can be written as

$$
W(t) \stackrel{\mathrm{d}}{=}(1-U) X(t)+U\left\{X(t)+Y_{0}(t)\right\}
$$

where $X(t)+Y_{0}(t) \stackrel{\mathrm{d}}{=} Y$, independent of $t$; compare Corollary 3.3.

\section{Analogues for lattice distributions}

In this section we briefly present the analogues of Theorems $3.1,4.1$ and 4.2 for $\mathbb{Z}_{+}$-valued random variables $X$. We consider the discrete-time renewal process generated by $X$ with $\mathbb{P}(X=k)=p_{k}$ for $k \in \mathbb{Z}_{+}$and $\mathbb{E} X=\mu \in(0, \infty)$, and use the 
notation as established in Lemma 2.2. We restrict attention to $X$ with $p_{0}>0$. This leads to the clearest analogues, and it is no real restriction: The results for $Z$ hold for arbitrary $X$; for the result on $W$ the restriction $\mathbb{P}(X \leq 1)>0$ is necessary, and the case $p_{0}=0, p_{1}>0$ can be reduced to the case $p_{0}>0$ by a shift. The proofs differ only slightly from those in Sections 3 and 4 .

Theorem 5.1. Let $X$ have an aperiodic distribution $\left(p_{k}\right)_{k \in \mathbb{Z}_{+}}$on $\mathbb{Z}_{+}$with $p_{0}>0$. Then the random variable $Z$ with distribution given by (2.4) satisfies

$$
Z \stackrel{\mathrm{d}}{=} X+Y
$$

where $Y$ is a (necessarily $\mathbb{N}$-valued) random variable independent of $X$, iff $X$ is infinitely divisible.

Proof. By (2.4), saying that $Z$ satisfies (5.1) with $X$ and $Y$ independent, is equivalent to the following assertion:

$$
\mathbb{P}(Z-1=k)=\frac{1}{\mu}(k+1) p_{k+1}=\sum_{j=0}^{k} p_{j} \mathbb{P}(Y-1=k-j) \quad\left[k \in \mathbb{Z}_{+}\right] .
$$

An appeal to Lemma 2.7 finishes the proof; note that necessarily $\sum_{k=0}^{\infty} r_{k}=\mu$.

From the proof it will be clear that $Y$ in (5.1) has distribution $\left(r_{k-1} / \mu\right)_{k \in \mathrm{N}}$. Of course, also Theorem 3.2 has a lattice analogue; we don't spell it out.

The analogues of Theorems 4.1 and 4.2 are combined in the following theorem. Note that a compound-exponential distribution on $\mathbb{Z}_{+}$necessarily has positive mass at 0 .

Theorem 5.2. Let $X$ have an aperiodic, compound-exponential distribution $\left(p_{k}\right)$ on $\mathbb{Z}_{+}$. Then the random variable $Z$ with distribution given by (2.4) satisfies

$$
Z \stackrel{\mathrm{d}}{=} X+X^{\prime}+Y_{0}
$$

where $X^{\prime} \stackrel{\mathrm{d}}{=} X, Y_{0}$ is $\mathbb{N}$-valued, and $X, X^{\prime}$ and $Y_{0}$ are independent. For the random variable $W$ with distribution given by (2.5) one has

$$
W \stackrel{\mathrm{d}}{=} X+A
$$

where $X$ and $A$ are independent and $A$ has a nonincreasing distribution on $\mathbb{N}$.

Proof. By Lemma 2.8 the pgf $P$ of $X$ has the form $P=1 /(1-\log Q)$ with $Q$ an infinitely divisible pgf; hence differentiation of $1 / P$ leads to the equation $P^{\prime} / P^{2}=Q^{\prime} / Q$. Since the pgf $P_{Z-1}$ of $Z-1$ is given by $(1 / \mu) P^{\prime}$, it follows that

$$
P_{Z-1}(z)=\{P(z)\}^{2}(1 / \mu) Q^{\prime}(z) / Q(z)
$$


Now, $(1 / \mu) Q^{\prime} / Q$ can be read as the pgf of $Y_{0}-1$ for some $\mathbb{N}$-valued random variable $Y_{0}$; cf. the remark following Theorem 5.1. We conclude that $Z$ satisfies (5.2). With regard to (5.3) it is sufficient to note that

$$
P_{W-1}(z)=\frac{1-P(z)}{\mu(1-z)}=P(z) \frac{-\log Q(z)}{\mu(1-z)}=P(z) \frac{1-G(z)}{(\mu / \alpha)(1-z)} ;
$$

cf. the second representation of (2.13) in Lemma 2.8 .

\section{An example}

For $X(\cdot)$ in Theorem 3.2 we take a Gamma process: $X(t)$ has a gamma distribution with parameters $t$ and $\lambda$, so with density $x \mapsto \lambda^{t} x^{t-1} \mathrm{e}^{-\lambda x} / \Gamma(t)$. The canonical function of $X(t)$ has density $x \mapsto t \mathrm{e}^{-\lambda x}$, and it follows that for the total life-time $Z(t)$ in the stationary renewal process generated by $X(t)[t>0$, fixed] we have

$$
Z(t) \stackrel{\mathrm{d}}{=} X(t)+Y
$$

where $X(t)$ and $Y$ are independent and $Y$ has an exponential distribution with parameter $\lambda$, independent of $t$.

For $0<t \leq 1$ the random variable $X(t)$ is compound-exponential: its LSt can be represented as $1 /\left(1-\log \widehat{F}_{0}\right)$, where $F_{0}$ is infinitely divisible with canonical function $K_{0}$ satisfying

$$
\widehat{K}_{0}(s)=-\frac{\widehat{F}_{0}^{\prime}(s)}{\widehat{F}_{0}(s)}=-\frac{\mathrm{d}}{\mathrm{d} s} \log \widehat{F}_{0}(s)=\frac{t}{\lambda}\left(\frac{\lambda}{\lambda+s}\right)^{1-t} .
$$

From Theorem 4.1 it follows that for $0<t \leq 1$

$$
Z(t) \stackrel{\mathrm{d}}{=} X(t)+X^{\prime}(t)+Y_{0}(t)
$$

where $Y_{0}(t)$ has a gamma distribution with parameters $1-t$ and $\lambda$. The special case $t=1$, i.e., $X(1)$ exponential, gives $Y_{0}(1)=0$ with probability 1 , as is well known. In general, $Z(t)$ has a gamma distribution with shape parameter $t+t+(1-t)=t+1$; for $t=\frac{1}{2}$ the random variable $Z(t)$ is the sum of three iid random variables.

By Theorem 4.2, for the remaining life-time $W(t)$ in the renewal process generated by $X(t)$ we find

$$
W(t) \stackrel{\mathrm{d}}{=} X(t)+A(t)
$$

with

$$
\widehat{F}_{A(t)}(s)=\frac{(1+s / \lambda)^{t}-1}{t s / \lambda} .
$$

For $t=1$ we obtain the well-known result that $W(1) \stackrel{\text { d }}{=} X(1)$, i.e., $\mathbb{P}(A(1))=0)=1$. 


\section{References}

[1] FELlER, W. (1968). An introduction to probability theory and its applications, vol. 1, 3-rd ed., Wiley, New York.

[2] Feller, W.(1971). An introduction to probability theory and its applications, vol. 2, 2-nd ed., Wiley, New York.

[3] HARN, K. VAN (1978). Classifying infinitely divisible distributions by functional equations, Math. Centre Tracts 103, Math. Centre, Amsterdam.

[4] Ross, S.M.(1970). Applied probability models with optimization applications, Holden-Day, San Francisco.

[5] Steutel, F.W. (1970). Preservation of infinite divisibility under mixing, and related topics, Math. Centre Tracts 33, Math. Centre, Amsterdam.

[6] Winter, B.B. (1989). Joint similation of backward and forward recurrence times in a renewal process, J. Appl. Prob. 26, 404-407.

July 15, 1994 